\section{Spezifische Immuntherapie - Erfahrungen in der Onkologie}

\author{
M. Gramatzki ${ }^{1}$ \\ 1 Ärztl. Leiter, Sektion für Stammzell- und Immuntherapie, \\ II. Medizinische Klinik, Dr. Mildred Scheel Haus, \\ Universitätsklinikum Schleswig-Holstein
}

Es wird intensiv an der Frage gearbeitet, wie mit den Waffen des hochspezifischen Immunsystems nach Chirurgie, Chemooder Strahlentherapie residuelle Tumorzellen vernichtet werden können. Dabei wurden einzelne Immuntherapiestrategien schon vor über 100 Jahren formuliert, etwa der Antikörpereinsatz durch Paul Ehrlich. Aber erst die durch Georges Köhler und César Milstein entwickelte Zellfusionstechnologie erlaubte, monoklonale Antikörper von prädefinierter Spezifität in praktisch unbegrenzter Menge herzustellen. Für die breitere klinische Anwendung war zudem eine Innovation in der pharmazeutischen Herstellung, die Biotechnologie, essenziell.

Sowohl für Autoimmunerkrankungen (AID) wie auch zur Tumortherapie sind in den 1990er Jahren monoklonale Antikörper erstmals breiter klinisch eingesetzt worden. In der Onkologie wurde sowohl mit Rituximab für die Eradikation von B-Lymphozyten bei Lymphomen wie auch mit Trastuzumab zur Abtötung Her2/neu überexprimierender Mammakarzinomzellen zwei erfolgreiche Therapeutika eingeführt. Das sehr akzeptable Nebenwirkungsprofil machte zudem eine Kombination mit Chemotherapie für optimale Behandlungsergebnisse bei beiden Antikörpern leicht möglich. Der später auch bei AID eingesetzte CD20-Antikörper Rituximab ist ein gutes Beispiel für die Wirkung eines Antikörpers gegen B-Lymphozyten, der B-Zellen depletiert, aber mit unterschiedlicher Intention eingesetzt wird. Auch der CD52-Antikörper Alemtuzumab kann unterschiedlich in dem jeweiligen Therapiefeld indiziert sein, nämlich einerseits zur Eradikation von B- und T-Zellleukämien und andererseits zur Immunsuppression bei und nach Transplantationen, wie etwa bei der lebensgefährlichen Transplantat-gegen-Wirt-Erkrankung (GvHD) nach allogener hämatopoietischer Stammzelltransplantation.

Während Antikörper gegen Oberflächenstrukturen auf Immunzellen früh entwickelt wurden, war das Auffinden Tumor-typischer oder -assoziierter Strukturen für andere, insbesondere solide Tumoren schwieriger. Auch für das Multiple Myelom gab es lange keinen therapeutischen Antikörper, bis kürzlich gleich zwei neue für die Antigene CD38 und CD319 erfolgreich getestet wurden. Zudem wird derzeit intensiv an besser Immunzellen aktivierenden Antikörperformaten gearbeitet und auch an Immunkonstrukten. Bei einer Form der Immunkonstrukte, den sogenannten AntibodyDrug-Conjugates (ADC), wird ein Toxin an einen Antikörper gekoppelt. Dies erlaubt, spezifisch in die Tumorzellen toxische Substanzen zu bringen, und vermeidet Nebenwirkungen an vielen anderen Organen. Solche ADC sind bereits klinisch etabliert, etwa mit Brentuximab Vedotin für den Morbus Hodgkin und CD30-positive T-Non-Hodgkin Lymphome oder mit Trastuzumab Emtansin (T-DM1) beim Her2/neu-positiven Mammakarzinom.

Aber auch ohne Toxinanbindung können durch Antikörperengineering heute vielfältige Antikörperkonstrukte hergestellt werden, die u.a. in Molekülgröße und Bindungsverhalten deutlich optimiert sind. So gelingt es, durch Engineering von Karbohydrat- oder Proteinkomponenten im Fc-Teil des Antikörpers die Bindung an die immunologische Effektorzelle und damit die antikörpervermittelte Zytotoxizität massiv zu erhöhen. Bi- oder tri-spezifische Antikörperformate erlauben ebenfalls eine verbesserte Tumorzellabtötung. Mit solch einem Format können auch andere Rezeptoren und auch auf anderen Effektorzellpopulationen, wie etwa T-Lymphozyten, direkt angesprochen werden. Das von Gert Riethmüller und Mitarbeitern wesentlich entwickelte BiTE-Konzept erlaubt etwa, T-Lymphozyten über CD3 massiv zu aktivieren. Aber nicht nur für die direkte Tumorzellabtötung sind Antikörper entwickelt worden, sondern sowohl für AID als auch für Tumoren werden Antikörper eingesetzt, die in Zytokinnetzwerke eingreifen (z.B. gegen Tumornekrosefaktor (TNF), Vascular endothelial growth factor (VEGF) oder Interleukin (IL)-6).

Zelluläre Immunmechanismen mögen je nach Krankheitsaktivität in ihrer Ausprägung als zu stark oder zu schwach empfunden werden; ihre Spezifität und Potenz ist jedenfalls erheblich. Im Rahmen der allogenen hämatopoietischen Stammzelltransplantation wird die Zielgenauigkeit und Nachhaltigkeit des Immunsystems immer wieder eindrücklich unterstrichen. Bei Rezidiven von Leukämien und Lymphomen nach SCT können Spenderlymphozyteninfusionen (DLI) zur erneuten Remission führen. Allerdings war aufgrund ihrer Komplexität die schon lange explorierte adoptive T-Zell-Therapie gegen Tumoren, wie etwa mit der Expansion von Tumor-Infiltrating-Lymphocytes (TIL), nur in wenigen Situationen erfolgreich. Dies hat sich in den letzten Jahren insbesondere durch die Arbeitsgruppe von Carl June geändert: Durch mit einem tumorgerichteten Antigenrezeptor modifizierte T-Lymphozyten, also sog. Chimeric-Antigen-Receptor (CAR) T-cells, wurden beeindruckende Tumorkontrollen in austherapierten Leukämiepatienten erreicht. Interessanterweise scheint ein gewisser Zytokinsturm dabei mit dem Ansprechen zu korrelieren. Es bleibt abzuwarten, ob CAR-TLymphozyten oder optimierte Antikörperkonstrukte in einer jeweiligen Tumorsituation effektiver sind.

In den letzten Jahren ist es in der Onkologie mit Hilfe von Antikörpern gegen die immunregulatorischen CheckpointMoleküle CTLA-4, PD-1 und PD-L1 gelungen, Blockaden des Immunsystems in der Tumorabwehr zu durchbrechen. In beeindruckender Weise konnte so eine Modulation des Immunsystems zur Bekämpfung bereits etablierter Tumoren erreicht werden. Nicht nur das Melanom, sondern auch andere Tumorentitäten, die bislang immunologisch inert waren wie HNO-, Blasen- oder Bronchialkarzinom, sprachen massiv an. Mittlerweile liegen für eine Kombination aus CTLA-4 und PD-1 Antikörpern, nämlich Ipilimumab und Nivolumab sogar Phase III-Daten vor, die in der Kombination noch bessere Therapieerfolge zeigen. Für die zelluläre Immuntherapie eröffnen sich durch die Beeinflussung solcher immunologischen Checkpoints völlig neue Perspektiven. 
Die Kenntnisse um solche immunregulatorischen Moleküle werden auch für Patienten nach Transplantationen oder mit AID wichtig sein, selbst wenn es hier vorrangig um die Dämpfung körpereigener Immunreaktionen geht. Da bei AID und Tumorerkrankungen ähnliche immunologische Effektorzellen und Steuerkreise beeinflusst werden und teilweise gleiche Therapeutika mit unterschiedlicher Intention eingesetzt werden, sollte der Austausch über Möglichkeiten und Grenzen solcher Therapiestrategien beide Forschungsfelder gegenseitig befruchten.

Interessenkonflikt: Kein Interessenkonflikt

\section{Bibliografie}

DOI http://dx.doi.org/10.1055/s-0035-1558069

Drug Res 2015; 65, Suppl. 1: S21-S22

(c) Georg Thieme Verlag KG Stuttgart · New York .

ISSN 2194-9379

\section{Korrespondenzadresse}

Prof. Dr. Martin Gramatzki

Ärztl. Leiter

Sektion für Stammzell- und Immuntherapie, II. Medizinische Klinik

Dr. Mildred Scheel Haus

Universitätsklinikum Schleswig-Holstein

Schittenhelmstr. 12

24105 Kiel

m.gramatzki@med2.uni-kiel.de

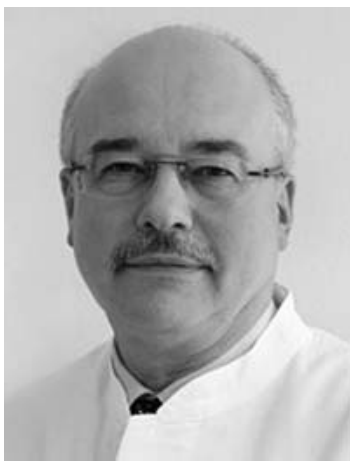

